\title{
The Future of X-Ray Time-Domain Surveys
}

\author{
Daryl Haggard ${ }^{1}$ and Gregory R. Sivakoff ${ }^{2}$ \\ ${ }^{1}$ Center for Interdisciplinary Exploration and Research in Astrophysics (CIERA), \\ Department of Physics and Astronomy, Northwestern University, Evanston, IL USA \\ email: dhaggard@northwestern.edu \\ ${ }^{2}$ Department of Physics, University of Alberta, Edmonton, AB, Canada
}

\begin{abstract}
Modern X-ray observatories yield unique insight into the astrophysical time domain. Each X-ray photon can be assigned an arrival time, an energy and a sky position, yielding sensitive, energy-dependent light curves and enabling time-resolved spectra down to millisecond time-scales. Combining those with multiple views of the same patch of sky (e.g., in the CHANDRA and XMM-NEwTON deep fields) so as to extend variability studies over longer baselines, the spectral timing capacity of X-ray observatories then stretch over 10 orders of magnitude at spatial resolutions of arcseconds, and 13 orders of magnitude at spatial resolutions of a degree. A wealth of high-energy time-domain data already exists, and indicates variability on timescales ranging from microseconds to years in a wide variety of objects, including numerous classes of AGN, high-energy phenomena at the Galactic centre, Galactic and extra-Galactic X-ray binaries, supernovæ, gamma-ray bursts, stellar flares, tidal disruption flares, and as-yet unknown X-ray variables. This workshop explored the potential of strategic X-ray surveys to probe a broad range of astrophysical sources and phenomena. Here we present the highlights, with an emphasis on the science topics and mission designs that will drive future discovery in the X-ray time domain.
\end{abstract}

Keywords. accretion, accretion disks, stars: flare, (stars:) novæ, cataclysmic variables, (stars:) supernovæ: general, stars: winds, outflows, galaxies: active, galaxies: nuclei, X-rays: general

\section{X-ray Astronomy's Broad Reach}

$\mathrm{X}$-ray data span an enormous dynamic range within astrophysical régimes. In the coming decades X-ray observatories, in concert with instruments across the electromagnetic spectrum, will systematically tackle the exciting "time domain". They will have enough power to reveal the progenitors to gamma-ray burst (GRBs), to probe the physics behind supernova (SN) shock breakout, to identify and characterize tidal disruption events, to constrain models of the accretion physics in X-ray binaries (XRBs) and active galactic nuclei (AGN), and to determine the rates and driving mechanisms behind stellar flares and their impact on space weather - to name a few examples. X-ray detectors are unique in that every photon is time tagged, energy tagged, and assigned an accurate sky position. X-ray observations also cover time-scales from sub-millisecond to $\sim 40-50$ years, span orders of magnitude in spatial resolution, and achieve a decade in energy coverage with decent energy resolution. The result is sensitive, energy-dependent light curves and time-resolved spectroscopy for every target. A wealth of high-energy time-domain data already exist, from which variability on time-scales ranging from microseconds to years has already been identified in a wide variety of objects.

In this workshop we discussed the missions that would be optimal for discovering and characterizing X-ray transients and variables. We were motivated in part by the enormous interest that has been expressed by the astronomical community (and evidenced by this very Symposium) in optical and (more recently) radio transients. We felt that the X-ray community is not, at present, making a compelling case for the power of X-ray 
observatories which are optimized for time-domain studies (with a few notable exceptions such as RXTE and SWIFT). We hope that the guiding questions outlined below, together with those generated by workshop attendees, will bring into focus the kinds of efforts needed to lobby most effectively for those missions, archives, cadences and science objectives in order to ensure that X-ray astronomy is well resourced in the future and thus able to contribute substantially to the exploration of transient phenomena.

The time domain is already expanding rapidly. To optimize these many transient domain studies we must connect the targets and the science at multiple wavelengths. Competition can be inimical to progress; although some might fear lest the optical and radio communities absorb resources away from the X-ray transient community, there was general consensus that the deepest insights into physics, and hence the highest science impact, result from coordinated, multiwavelength observations.

This overview of the workshop does not explore the full scope of science accessible in the X-ray domain, nor advocate any particular mission. Both the science and the technology are rapidly evolving, and attempts to place the entirety of X-ray astronomy under a single umbrella may be a questionable exercise - as explained in Martin Elvis' response to NASA's recent call for "Concepts for the Next NASA X-ray Astronomy Mission" $\dagger$. Instead, we hope to prompt the astronomical community into thinking about the central role which X-rays have played and still can and should play, in our exploration of astronomy's time domain.

\section{Guiding Questions}

We asked workshop attendees to discuss these guiding questions:

(a) In recent years optical and radio transient science have increasingly gained attention among the general astronomical community. At the same time, X-ray transient surveys seem to be ceding ground, both financially and scientifically. What are the most compelling science cases for current and future X-ray transient studies? What efforts does the X-ray transient community need to undertake to lobby most effectively for the importance of X-ray transient studies (past and present) to the general astronomical community?

(b) The Rossi X-ray Timing Explorer (RXTE) has been a tremendous boon for studies of X-ray transients. However, it will cease operation at the end of this year. While some of its scientific capacities can be shifted to current instruments like SWIFT and MAXI, other capacities are unique to RXTE among currently flown instruments. What steps do we need to take to transition from the era of RXTE to the era without it? What important lessons have we learned from RXTE? How will new planned or soon-to-belaunched instruments support X-ray transient surveys? What inventive ways can we develop to utilize new instruments that may not have been designed originally for X-ray transient studies?

(c) The scientific output of X-ray transient surveys can be greatly increased through multi-wavelength observations. How do we best coordinate multi-wavelength observations, especially for X-ray transient surveys? Do we need to develop an X-ray Transient Network, or are existing infrastructures like the Gamma-ray Circular Network and the Astronomers Telegram sufficient? What cadences are needed to achieve various science

$\dagger$ NASA has recently solicited the community to suggest new X-ray mission concepts for advancing the goals of the Physics of the Cosmos (POC) programme (NASA RFI NNH11ZDA018L). These submissions are public, and are available on the POC webpage: http://pcos.gsfc.nasa.gov/studies/x-ray-mission-rfis.php. 
priorities at different wavelengths? Are there opportunities for "citizen science" with $\mathrm{X}$-ray transient surveys?

\section{Workshop Highlights}

The workshop was structured as a pure discussion - there were no formal science talks. Some of the most active discussions that took place are outlined below. A 1.5-hour audio recording of the workshop, together with a written transcript, are available at http://faculty.wcas.northwestern.edu/ dha724/xray_transients_2011/.

\subsection{X-ray Transients and Variables}

Our first discussion was of transients (unanticipated [dis]appearance or flaring) as opposed to variables (periodic or repeated fluctuations). Are X-ray studies more likely to uncover "variables" than "transients"? The majority opinion was that most X-ray variables were initially identified as transients (as is indeed the case with optical/radio transients), and that in most cases the distinction is driven by the detection limits of individual surveys. For example, on very deep optical data (to $\sim 28^{\text {th }}$ magnitude) one may begin to see progenitors of Type Ia supernovæ (which are themselves are probably variable) in addition to novæ, X-ray binaries and the like.

\subsection{The Science Case(s) for X-ray}

It is essential to state the most compelling science cases for current and future X-ray transient studies - to identify what is unique about the X-ray domain and why it should be compelling to fund an X-ray mission rather than a UV or IR one. Strong gravity and accretion physics are both areas to which the X-ray time domain brings a unique view. The most interesting individual science cases for X-ray time-domain studies included:

- Gamma-ray bursts (black-hole birth, cosmological probes)

- Supernova shock break-out

- Tidal disruption events

- X-ray variability of AGN and XRBs

- Giant hard X-ray flares (from flare stars and blazars)

- Impact of stellar flares on space weather/planetary habitability

- Variability in SgrA*

- Accreting millisecond pulsars

- Coherent pulsations and QPOs in neutron stars

- Galactic black-hole and neutron-star populations

In addition, other X-ray variables, not yet recognized as such, might supply the most compelling physical insights, though it is difficult if not impossible to base an X-ray mission on only an anticipated benefit. Many of the phenomena cited were originally discovered in the X-ray domain (though most remain only poorly characterized). However it was felt that, in coming years, the impetus will most likely come not from X-ray missions but from optical or radio telescopes, reflecting an enthusiasm for the "new" (LOFAR, ATA, PTF and potentially LSST) as opposed to the "old" or established (RXTE All-Sky Monitor, SwIFT, XMM-NEwTON and Chandra surveys). If we make out that the X-ray sky is a known entity, then the potential for discovery is perceived to be greater at less known wavelengths, making the latter seem more exciting. An X-ray transient mission therefore needs some goal like testing general relatively to bolster its case, i.e., something that can only be done through X-ray science. 
X-ray variability in AGN and XRBs probes the physics of the inner accretion disk. These, in particular, test strong gravity. The same is true for tidal disruption events. The structure of the variability and its time-scale may assist in distinguishing between radiatively efficient and inefficient accretion flows and the mechanisms responsible for launching jets and winds. Sensitivity to very rapid variations (coherent pulsations, QPOs, fractional variability) is critical for understanding local XRB sources, and may shed light on more distant sources by analogy.

From the multiwavelength perspective, radio quenching and radio flaring have been seen in X-ray binaries within days. Hence, having missions that have the capacity to observe an XRB daily after an outburst has proved critical. The difficulty now is coordinating efficiently with other observatories; in the radio (for example) coordinations with EVLA have improved with the introduction of dynamic scheduling, but are still of the order of a few hours.

For AGN the relevant time-scale is weeks to years. As pointed out, AGN go into a deep low state and stay there for days or weeks; that is when the X-ray spectral complexity is most pronounced, and when distinguishing between different inner disk models is most effective. Thus, for AGN it might be the dips in their light curves, not the flares, which prove more interesting. Monitoring tidal disruption flares is also most effective on timescales of weeks, but time-scales of minutes have not yet been explored for blazars. At GeV energies we are limited by statistics, but there is sub-day variability, and presumably it is the X-ray non-thermal component that is varying. As described by Kulkarni (p. 55), the time-scale for the X-ray shock break-out from supernovæ is hours or less.

In stellar coronal variability, both sensitivity and wavelength coverage are important. Greater sensitivity allows one to look for flares from stars at greater distances or for weaker flares from stars less distant, but we require the multi-wavelength context (soft $\mathrm{X}$-rays, hard X-rays, UV/optical) to facilitate a full interpretation. X-ray emission from stars provides information about the coronal material and the coronal dynamics, and which cannot be obtained from other wavelengths; X-rays show how the tenuous coronal plasma is reacting to magnetic reconnection. There are many aspects regarding stellar flares that are of outstanding interest. What drives the extreme energy release? How do

\section{Prospects for X-ray Time Domain Surveys}

\begin{tabular}{|c|c|c|}
\hline $\begin{array}{l}\text { Discovery \& } \\
\text { Monitoring }\end{array}$ & Rapid Response & High Time Resolution \\
\hline $\begin{array}{l}\text { - } \text { All Sky Monitor } \\
\text { - } \text { MAXI/AstroSAT, } \\
\text { Lobster-eye } \\
\text { technology, LOFT } \\
\text { - Science drivers: } \\
\text { GRBs, SNe shock } \\
\text { breakout, } \\
\text { accretion physics, } \\
\text { tidal disruptions } \\
\text { - Daily Cadence }\end{array}$ & $\begin{array}{l}\text { - } \text { Rapid slew (< hr) } \\
\text { - Swift, AstroSAT, } \\
\text { LOFT, a suite of } \\
\text { Swifts!? } \\
\text { - Science drivers: } \\
\text { GRBs, stellar } \\
\text { flares/space } \\
\text { weather, transient } \\
\text { response } \\
\text { - High Availability }\end{array}$ & $\begin{array}{l}\text { - Sub-ms timing } \\
\text { - AstroSAT, } \\
\text { Athena, } \\
\text { LOFT? } \\
\text { - Science drivers: } \\
\text { Strong gravity, } \\
\text { neutron star } \\
\text { physics, XRB } \\
\text { physics, QPOs } \\
\text { - High Sensitivity }\end{array}$ \\
\hline
\end{tabular}

Figure 1. 
flares affect the stellar environment (both in the context of young stars where planets are forming in a disk, and for older stars where planets have already formed)? How might flares affect habitability? Flares need to be understood in the context of larger magnetic processes, and dynamo processes. To study stellar flares in detail requires high timeresolution, e.g., responses within minutes, because most of the energy in the initial flare is released in the first few minutes in the so-called "impulsive" phase, when one expects to see hard X-rays and radio emission; later the flare transitions to the "gradual" phase when thermal X-rays and the UV/optical responses begin to dominate. Observations at different time-scales thus probe different physics. Statistics of stellar flares can usefully be derived on all times-scales: minutes, hours and days.

Another extension of stellar flare science involves space weather and the impacts upon the Earth. Studying the solar corona might in principle teach a great deal about flares on just one class of star but would teach little about its past and projected future behaviour; broadening the sample to many different types of star suggests how the Sun behaved in the past, and how it might behave in the future. The inverse of the argument is to regard observations of stars as proxies for modelling how the Sun's influence on space weather might evolve. Strength might then be given to potential new missions by opening them to other scientific communities (and their resources), though careful crafting of the science case would be imperative.

Clearly, the cadences required (minutes to years) depend crucially on the class of sources being explored. Different science goals are best accomplished with different technologies (see Fig. 1). Very fast transients represent territory that is largely unexplored, while at the other end of the scale all-sky X-ray monitoring programmes have mission lifetimes that are poorly matched to the long (rest-frame) variability time-scales of AGN. At present there is too much reliance upon serendipity; the 1999 flare of Sgr V4641, for instance, or the recently reported outburst in the Arches cluster could easily have been missed.

\subsection{Multi-wavelength Coordination}

At several junctures the workshop discussed practices for coordinating multi-wavelength observations and sending alerts to the community. For example, is there a need to develop an X-ray Transient Network, or are existing infrastructures like The Gamma-ray Circular Network and the Astronomer's Telegram (ATel) sufficient? (see p. 221). One existing problem is a degree of confusion in nomenclature. If different groups use different names to identify the same sources, it results in complications and leads to duplicate follow-up observations. This seems to be particularly true when the Galactic Centre is up and is being observed by InTEGRAL. A "transient wiki" could keep track of everything that is currently active. An increase in the number of joint proposals allowed (e.g., NASA+ESO or ESA+NOAO/Australian facilities) might also be important.

In general, ATels and other alert services seem to be serving the community well. Moreover, inside ATel there is now the AtelStream, which is a scheme for unifying announcements. There is also no doubt that the situation regarding joint proposals has improved tremendously over the past decade, but the need for a continued push for time-share agreements and joint proposal opportunities, especially for projects which require strictly simultaneous data, is strongly supported. In practice, it currently requires a significant commitment of time to coordinate a multi-wavelength campaign, possibly because of identifiable structural issues: very few observatories are set up for multi-wavelength collaborations. One successful example is the excellent inter-agreement between SAO/CHANDRA and NRAO/EVLA, within which it is quite straightforward to obtain simultaneous $\mathrm{X}$-ray and radio observations. Two modes are involved: the 
"discovery mode" for the transients, requiring rapid slew and other time-critical followups, and the "follow-up" mode when multiple instruments need to bear down on the same target. The latter mode requires either robotic streams or actual structural changes to the way in which time is granted and/or scheduled.

Multi-wavelength follow-up of X-ray targets can also suffer from a mismatch in timing resolution. For example, X-ray data are time-tagged, and events can be resolved easily at the millisecond level, but that information is of little help when trying to coordinate those data with an IR observation, where integrations run for minutes or longer, and the outcome is a comparison of two completely different time domains. One solution might be to use large-format optical/IR photon-counting detectors which automatically incorporate time tagging, discussed by O'Brien (p. 385) and Welsh (p. 99). Absolute timing stamps can also be incorporated. However, very high time-resolution detectors generate enormous quantities of data and huge files.

\subsection{Optimizing Existing and Future X-ray Missions for Time-Domain Science}

The workshop discussed the following past, present, and future X-ray missions in detail and how they might accomplish the science goals outlined above:

Planned/Proposed: NuSTAR, AstroSAT, ASTRO-H, eROSITA, GEMS, SVOM, Athena, LOFT, WFXT, JANUS, Lobster, Smart-X, also earlier footnote

Active (+Archival): Chandra, XMM-Newton, Suzaku, SwIFT, INTEGRAL, MAXI Archival: RXTE, ROSAT, Einstein

The recording and transcript include descriptions of individual missions; see also White (p. 159). The instruments which are now current are also providing extensive archives that will be particularly useful for time-domain studies involving longer baselines.

The relative merits of an X-ray all-sky monitor, rapid slew missions and missions or instruments optimized for high time-resolution came in for considerable discussion. There had been broad support at a HEAD meeting 11 years ago for an X-ray all-sky monitor, but as the demand for sensitivity increased the payload grew, and soon it faced much stiffer competition as a stand-alone mission - and lost. The landscape may be different now owing to the rapid growth of, and huge investment in, ground-based programmes like the Palomar Transit Factory (PTF) and Pan-STARRS, and the Large Synoptic Survey Telescope (LSST) promised in the next decade. When the radio equivalent is also added, the demand for X-ray all-sky sensitivity at least an order of magnitude better than present values will surely increase. Among the missions that might fill that niche are Janus and Lobster-eye detectors, described in White (p. 159).

In discussing the lessons learned from RXTE, the workshop recognised that flexibility in responding to target of opportunity requests $(\mathrm{ToO})$ is critical for X-ray timing studies, that the discipline needs a capability to observe how the timing properties themselves change in time (they are sharper probes than changes in the energy spectra), and that some of the work done by RXTE - in particular the all-sky monitoring - can be done in the optical and infrared from the ground because most X-ray binaries (except the highly extincted ones) show enhanced optical and infrared emission during outburst. SwIFT has been fantastic in its rapid response to ToOs and can take over nicely from RXTE in certain régimes, but lacks the effective area for RXTE's timing work, specifically for the study of pulsations and QPOs. It is possible that the Indian mission AstroSAT (the launch is planned in 2012) will recover many more of RXTE's capabilities, and may even improve on them through its increased sensitivity. The AstroSAT data will be proprietary, 
but the possiblity for real-time release of transients remains open. The data archive will be housed at the Inter-University Centre for Astronomy and Astrophysics (IUCAA), but the plans for access to the data are unclear.

Data access and availability of funding, particularly for serendipitous and archival studies, influence which science and which missions gain traction in the astronomical community. One drawback of mission designs like that of SwIFT is its lack of funding for scientists pursuing ToOs. A similar problem affects the many X-ray mission archives such as ROSAT, CHANDRA, XMM-NEWTON, etc., that could be used for transient and variability science, as well as the utilization of multi-wavelength archives like GALEX and SDSS. Unfortunately, since most archival research is funded through soft money, competition for that funding influences the type of science that gets done since proposals need to be tailored to the preferences of the funding agencies. One possible funding programme is NASA's Research Opportunities in Space and Earth Sciences programme, which funds research connected with NASA missions, including Fermi, Chandraand GALEX. Indeed, radio astronomers studying compact objects could access NASA monies to do the radio follow up. In the EVLA's model for data sharing, the so-called RSRO time (Resident Shared Risk Observing), an observing team could be awarded pre-commissioning time in return for at least one team expert taking "in residence" status at the facility, but such a programme is unlikely to be workable within a space-based context. However, pipeline and software development was proposed as one area where data exchange might be feasible.

Future advances are likely to require yet higher time resolution and higher energies. The proposed Large Observatory For X-ray Timing (LOFT; possible launch 2020) is a high-sensitivity time-domain mission that could be the sort of instrument required; one possible science driver could be the spectral timing of black holes and AGN. An alternative might be a vast improvement in "gamma-ray burst" type capabilities, such as an instrument with the solid angle of BAT but 10 times more sensitivity, better source localization, and with an IR telescope; it would open up a huge phase space which has never been probed before. Such a mission would specifically support a range of science projects, from SN shock break-outs and tidal disruptions to moderate redshift, gamma-ray bursts at $z>9$ and searches for the periods of ultra-luminous X-ray sources. Meanwhile, the Wide Field X-ray Telescope, a proposed medium-class NASA mission, could be a powerful instrument for transient detections in the distant universe and could consider targeting the LSST Deep-Drilling fields repeatedly during its lifetime.

\subsection{Opportunities for Citizen Science}

As a final topic for discourse, the workshop explored possibilities to involve citizen science in X-ray transient studies. So far there has been little involvement by non-specialists in Xray or high-energy programmes. However, citizen science is rapidly becoming recognised as a way of getting interesting science done and-more importantly - of engaging the public and also achieving certain tasks that need to be carried out in order to justify the investment in support of science.

It was felt that the amateur community who normally worked in the optical domain would be enthusiastic about following up X-ray transients. Help could be enlisted through a message to the American Association of Variable Star Observers (AAVSO) or similar organization, seeking observers willing to follow a 14,15 or $16^{\text {th }}$ magnitude object. Even though it is unlikely that the faintest targets could thus be followed, the benefit to X-ray science would be the adaptation of abundant capabilities across multiple wavelengths. Indeed, many of the AAVSO data are of exceptionally good quality, and amateur 
observers collectively have the advantage of wide longitudinal coverage, something which is not possible for many professional astronomers.

It should be recognized, however, that searches which make extensive use of existing data can be computationally intensive - for instance, if one tried to find every possible transient in the INTEGRAL or BAT archives, or looked for transients in the CHANDRA and XMM-NEWTON deep fields on every possible timescale.

Such archival searches are often very RAM intensive and may not be adaptable to software that runs on unused cycles in the same way that (say) SETI@home or Einstein@home can be run. Another suggestion was to coordinate amateurs to monitor dense regions of the sky in some systematic way in order to observe new X-ray binaries in outburst. Many of those systems rise quickly to $\sim 16^{\text {th }}$ magnitude, and nowadays that is within reach for a large number of amateurs.

\section{Summary}

The X-ray time domain uniquely probes strong gravity, accretion physics, supernova shock break-out and stellar flares. Specific tests of the first two involve the inner accretion disks of X-ray binaries and AGN. Changes in the X-ray variability and in its timescale probe the structure of accreting degenerate systems, and increased sensitivity to rapid variations enables studies of XRB pulsations, QPOs, and fractional variability. The spectral timing of black holes and AGN may also reveal the structure, and deep low states in AGN may give a particularly clean glimpse into their spectral complexity. Shock break-out has been an exciting topic that has featured throughout the Symposium, and the race is on for the first observations of an X-ray shock break-out. X-ray emission from stellar flares probe coronal material and its dynamics. Most of the energy from the initial flare appears in the X-ray and radio, usually within a few short minutes. Stellar and solar flares are critical to our understanding of space weather, and may have a profound impact on the habitability of planets.

\section{Acknowledgements}

We thank the 30 or so scientists who attended this workshop and contributed to our discussion, and in particular we thank Phil Charles, Stephane Corbel, Boris Gaensicke, Stefanie Komassa, Shri Kulkarni, Ashish Mahabal, Roberto Mignani, Rachel Osten, Danny Steeghs, Tom Vestrand, Barry Welsh, Peter Williams, and Patrick Woudt for generating lively dialogue. We also appreciate input from Niel Brandt, Craig Heinke, Tom Maccarone, and Richard Mushotzky, who commented on our guiding questions though they were not able to attend. 